\title{
Evaporation-driven assembly of colloidal particles
}

\author{
Eric Lauga \& Michael P. Brenner \\ Division of Engineering and Applied Sciences, Harvard University, \\ 29 Oxford Street, Cambridge, MA 02138.
}

(Dated: November 13, 2018)

\begin{abstract}
Colloidal particles absorbed at the interface of a liquid droplet arrange into unique packings during slow evaporation (Manoharan et al. Science 301 483-487). We present a numerical and theoretical analysis of the packing selection problem. The selection of a unique packing arises almost entirely from geometrical constraints during the drying.
\end{abstract}

Manoharan et al. 1] recently presented an ingenuous method for fabricating clusters of small particles into precise configurations. Polystyrene spheres (diameter 844 $\mathrm{nm}$ ) were dispersed in a toluene-water emulsion with each oil droplet containing a low number $N$ of spheres. The toluene was then preferentially evaporated, forcing the particles to come together into compact clusters. Surprisingly, the final particle packings were unique: the observed packings for $N \leq 11$ closely correspond to those previously identified 2] as minimizing the second moment of the particle distribution, $\mathcal{M}=\sum_{i}\left\|\mathbf{r}_{i}-\mathbf{r}_{0}\right\|^{2}$, where $\mathbf{r}_{0}$ is the center of mass of the cluster.

The fact that such a simple process leads to precision assembly at the submicron scale points to exciting possibilities for controlling the assembly of more general objects [3]. The goal of this Letter is to understand the physical principles underlying the observations of Manoharan et al. [1]. Why are the final packings unique? (Why) do they minimize the second moment? What physical parameters do these results depend upon? We first present numerical simulations of hard spheres on an evaporating liquid droplet for a wide range of liquidsolid contact angles: for each contact angle the simulations reproduce the final packings of 1]. We then demonstrate that the uniqueness of the packings, as well as their connection to minimal moment structures, can be understood from purely geometrical considerations. The arguments suggest a methodology for creating new packings, which we confirm through numerical simulations.

Numerical Simulations. The experiments suggest the following theoretical problem: for a given liquid volume, the particle configuration is determined from minimizing
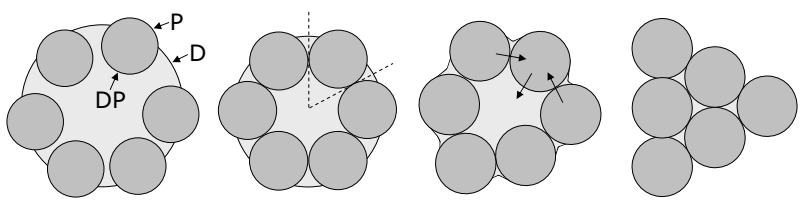

(a)

(b)

(c)

(d)

FIG. 1: Schematic two-dimensional representation of the drying experiment: (a) particle configuration when the droplet volume is above the critical volume; (b) critical packing; (c) rearrangement below the critical packing with both capillary and contact forces acting on each particle; (d) final packing. the total surface energy

$$
U_{\Sigma}=\gamma_{D} \int_{D} \mathrm{~d} S+\gamma_{D P} \int_{D P} \mathrm{~d} S+\gamma_{P} \int_{P} \mathrm{~d} S
$$

while respecting excluded volume constraints between the particles. Here $D, D P$ and $P$ refer to the droplet surface, droplet-particle interface and particle surface respectively (see Figure 1).

Numerical simulations of this problem are performed using Brakke's Surface Evolver [4], a program which determines the equilibrium configuration of deformable surfaces given the definition of an energy. The colloidal spheres are modelled as liquid droplets with high surface tension, typically 1 to 2 orders of magnitude larger than the main droplet, in order to penalize non-spherical deformations of their shape. Interfacial tension between the droplet and the particles are chosen appropriately in order to satisfy Young's law at the solid-liquid contact line, $\gamma_{P}=\gamma_{D} \cos \theta+\gamma_{D P}$, where $\theta$ is the equilibrium contact angle. Non-interpenetrability is enforced with an excluded volume repulsion energy $U_{R}$ acting between the centers of the spheres [12]; $U_{R}$ dominates when at least two spheres overlap by one percent.

The particles are initially positioned randomly on the droplet. The droplet volume is then slightly decreased (by one percent or less) and the particles rearrange to a new equilibrium, minimum of $\mathcal{U}=U_{\Sigma}+U_{R}$. This procedure replaces the evaporation dynamics by a series of equilibrium problems and therefore mimics the low evaporation rate limit of the experiments.

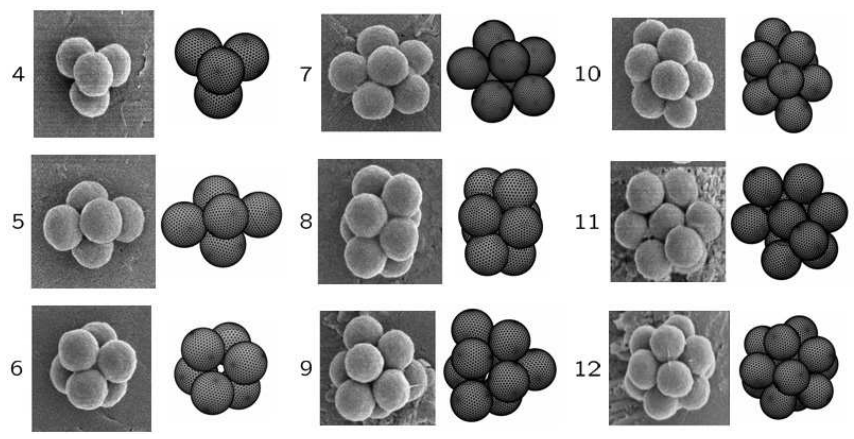

FIG. 2: Comparison of the experimentally observed packings (left) with those obtained by numerical simulations (right) as a function of the number of spherical particles. 


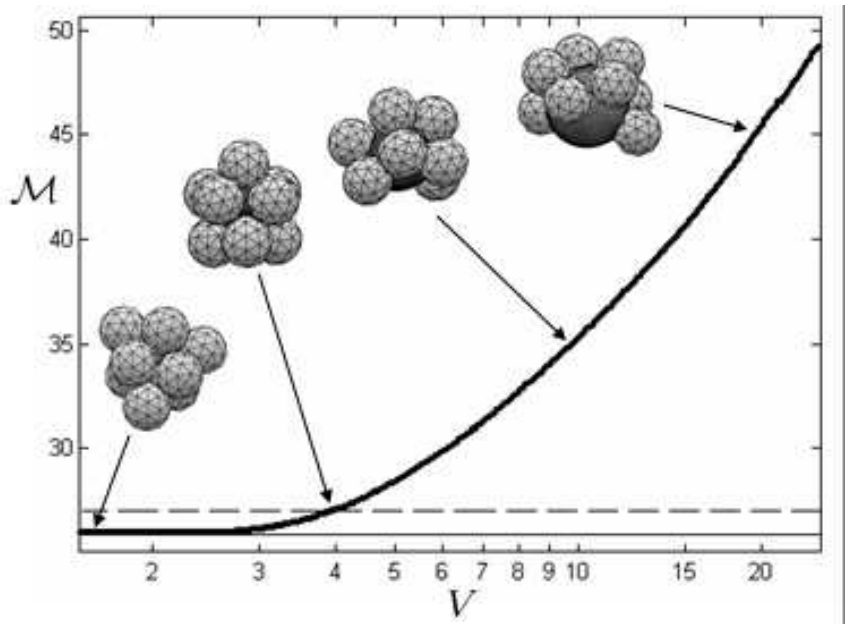

FIG. 3: Numerical evolution of the second moment as a function of the droplet volume for $N=9$ (both in units of the particle radius). The final second moments are 25.899 (theory) and 25.946 (simulations). Insert: packings observed during the drying process when $\mathcal{M}=25.946,27.016,35.014,45.058$. Solid line: minimal second moment; dashed line: second moment at the critical packing $(\mathcal{M}=27)$.

We find that the packings obtained numerically are unique and agree with those obtained by Manoharan et al. 1], over the range of contact angles tested $\left(10^{\circ} \leq \theta \leq\right.$ $170^{\circ}$ ) and initial conditions. A comparison of the final computational and experimental packings is illustrated in Figure 2 In all cases, the final second moment obtained numerically differs by less than $0.5 \%$ from the minimum moment packings of [2]. The agreement verifies that the experimental packing problem corresponds to the energy minimization posed above and that other particleparticle interactions (electrostatic, van der Waals...) are not essential to generate the final packings.

There is one noteworthy difference between the simulations and the experiments. In the simulations, the cluster of spheres evolves smoothly and continuously throughout the drying process (c.f. Fig. 3 for 9 particles). In contrast, experiments [1] showed a discontinuous transition to the final packing, with an abrupt change in the second moment at a critical volume. The experimental discontinuities must arise from experimental features not present in the simulations, probably contact angle hysteresis.

The Critical Volume. We now study theoretically the packing selection problem. For sufficiently large liquid volumes, the minimum energy solution is a spherical droplet with noninteracting force-free particles (Fig. 17). There exists a critical volume $V_{c}$ below which the droplet cannot remain spherical (Fig. 10); below this critical volume each particle is acted upon by capillary forces. At the critical volume $V_{c}$, there is a critical packing of particles, which can be characterized as follows.

The interactions between particles on the surface of a sphere are equivalent to the steric interactions between the "cone of influence" of each particle, defined as the cone originating from the droplet center and tangent to

\begin{tabular}{|c|c|c|c|c|c|c|c|c|c|c|}
\hline$N n_{c}$ & $n_{f}$ & $n_{m}$ & $N$ & $n_{c}$ & $n_{f}$ & $n_{m}$ & $N$ & $n_{c}$ & $n_{f}$ & $n_{m}$ \\
\hline 46 & 1 & 1 & 13 & 24 & 1 & 1 & 21 & 40 & 1 & 1 \\
\hline 56 & 1 & 3 & 14 & 28 & 3 & 1 & 22 & 42 & 1 & 1 \\
\hline 612 & 3 & 1 & $15 a$ & 30 & 3 & 1 & 23 & 43 & 2 & 3 \\
\hline 712 & 1 & 1 & $15 \mathrm{~b}$ & 30 & 3 & 1 & 24 & 60 & 15 & 1 \\
\hline 816 & 3 & 1 & 16 & 32 & 3 & 1 & 25 & 48 & 1 & 1 \\
\hline $\begin{array}{ll}9 & 18\end{array}$ & 3 & 1 & 17 & 34 & 3 & 1 & 26 & 46 & 1 & 5 \\
\hline 1019 & 2 & 1 & 18 & 34 & 1 & 1 & 27 & 52 & 1 & 1 \\
\hline 1125 & 6 & 1 & 19 & 34 & 1 & 3 & 28 & 52 & 1 & 3 \\
\hline 1230 & 9 & 1 & 20 & 39 & 6 & 5 & 29 & 54 & 1 & 3 \\
\hline
\end{tabular}

TABLE I: Characteristics of critical packings of spherical colloidal particles as a function of their number $N$ : number of contacts $\left(n_{c}\right)$, number of independent forces $\left(n_{f}\right)$ and number of admissible modes of rearrangement $\left(n_{m}\right)$.

the particle (Fig. 10). These are also equivalent to the interactions between the intersection of these cones with the droplet, which are circles. Consequently, packing spherical particles at the critical volume is equivalent to packing circles on a sphere, a mathematical problem with rich history [5, 6, 7, 8] and for which numerical solutions have been proposed up to $N=\mathcal{O}(100)$. Usually, the circle packings for a given $N$ are unique, with two types of exceptions. For $N=5,19,20,23,26,28,29 \ldots$ the circle packing has continuous degrees of freedom (where at least one circle is free to "rattle") 8]; for $N=15$ two different configurations (15a and 15b) lead to the densest packings with equal surface density 8].

Hence, provided that there are no kinetic traps, identical particles will arrange themselves into the circle packing at the critical volume. Fig. 3 confirms this conclusion in numerical simulations for $N=9$. Note that changing the contact angle of a particle changes the size of its circle of influence and the droplet radius by the same amount, so the circle packing problem is unchanged and the critical packing of particles at the critical volume is independent of wetting characteristics; note however that the critical droplet volume is contact angle dependent.

How do the particles rearrange when $V<V_{c}$ ? The energy minimization problem suggests that we must find the particle configuration which minimizes $U_{\Sigma}$ under inter-penetrability and contact angle constraints. Owing to the complexity of solving for the liquid surface of constant mean curvature, this at first appears to be an extraordinarily difficult theoretical problem. However, we have found that in fact the constraints associated with packing of particles are sufficient to uniquely determine the initial rearrangements of the particles.

Let us suppose the droplet volume is reduced by a small amount $\delta V \ll V_{c}$. Deviations of the droplet interface from spherical lead to capillary forces $\left(\mathbf{F}_{i}\right)$ on each particle. Since every particle must be in force equilibrium, these forces must be balanced by contact forces $\left(\mathbf{f}_{j i}\right)$ between the particles (Figure 1:):

$$
\mathbf{F}_{i}+\sum_{j \in \mathcal{C}(i)} \mathbf{f}_{j i}=\mathbf{0},
$$




\begin{tabular}{llllllll}
$N$ & $\mathcal{M}_{\exp }$ & $\mathcal{M}_{m}$ & $\mathcal{M}_{2}$ & $N$ & $\mathcal{M}_{\exp }$ & $\mathcal{M}_{m}$ & $\mathcal{M}_{2}$ \\
\hline $\mathbf{3}$ & 4 & 4 & 4 & $\mathbf{9}$ & 25.899 & 25.899 & 25.899 \\
$\mathbf{4}$ & 6 & 6 & 6 & $\mathbf{1 0}$ & 31.828 & 31.828 & 31.828 \\
$\mathbf{5}$ & 9.333 & 9.333 & 9.333 & $\mathbf{1 1}$ & 37.835 & 37.929 & 37.835 \\
$\mathbf{6}$ & 12 & 12 & 12 & $\mathbf{1 2}$ & 43.416 & 43.416 & 42.816 \\
$\mathbf{7}$ & 16.683 & 17.100 & 16.683 & $\mathbf{1 3}$ & 51.316 & 52.690 & 47.701 \\
$\mathbf{8}$ & 21.157 & 21.657 & 21.157 & $\mathbf{1 4}$ & 59.225 & 60.279 & 54.878
\end{tabular}

TABLE II: Final second moment of the model $\left(\mathcal{M}_{m}\right)$ compared with the final second moment in the experiment [1] if the particles are assumed to be perfectly spherical $\left(\mathcal{M}_{\exp }\right)$ and with the minimum second moment $\left(\mathcal{M}_{2}\right)$.

where $\mathcal{C}(i)$ denotes the set of particles in contact with sphere $i$.

Let us characterize the number of ways the particles can rearrange to accommodate this change in volume. Each particle has three degrees of freedom and the droplet has one (the value of its mean curvature or pressure), so there are $3 N+1$ degrees of freedom. The constraints are of three types: (a) solid body rotation does not modify the packing (3 constraints); (b) the particles cannot overlap, ( $n_{c}$ constraints, where $n_{c}$ is the number of contacts at the critical packing); (c) forces have to balance (Eqn. 2).

Equation (2) implies nontrivial constraints. Indeed, suppose we try to solve (2) for the $n_{c}$ contact forces $\mathbf{f}_{j i}=f_{j i} \mathbf{e}_{j i}\left(\mathbf{e}_{j i}=\mathbf{e}_{i}-\mathbf{e}_{j}\right.$, where $\mathbf{e}_{i}$ is the unit vector directed from the droplet center to the center of the particle). Equation (2) has $3 N$ components, and $n_{c} \sim 2 N$ unknowns (Table I). Consequently, solutions exist only if compatibility relations are satisfied between the $\mathbf{F}_{i}$. Since capillary forces depend on the position of the particles, these equilibrium considerations constrain the rearrangement of the particles. Geometrical constraints have also played an important role in understanding force propagation in granular packings 9 .

Close to the critical volume, the capillary forces are given by $\mathbf{F}_{i}=F_{i} \mathbf{e}_{i}$. From the $N$ scalar forces $F_{i}$, equation (2) shows that only a subset $n_{f}$ can be chosen independently, i.e. equilibrium of each particle lead to $N-n_{f}$ additional constraints; $n_{f}$ is found by computing the rank of the compatibility matrix in (2) [13] .

The total number of admissible modes of rearrangement $n_{m}$ for the colloidal particles is found by subtracting the number of constraints from the number of degrees of freedom: we find $n_{m}=2 N-2+n_{f}-n_{c}$. For a given $N$, $n_{m}$ is entirely determined by the geometry of the critical packing. The results are displayed in Table @ Whenever the circle packing is unique, we find that $n_{m}=1$. When $n_{m}>1$, we find that the number of modes is always correlated with the presence of rattlers. If a total number of continuous degrees of freedom $n_{d}$ exist in the circle packing $\left(n_{d}=2\right.$ or 1 per rattler depending on if it completely free, as in $N=19$ or constrained in a slot, as in $N=5$ ), we always find that $n_{m}=1+n_{d}$. Since $n_{d} \neq 0$ indicates that there exists $n_{d}$ force-free surface

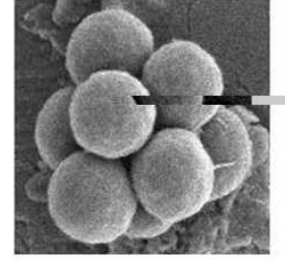

(a)

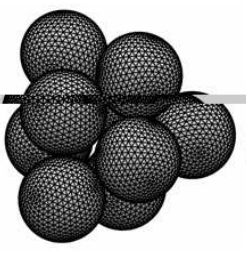

(b)

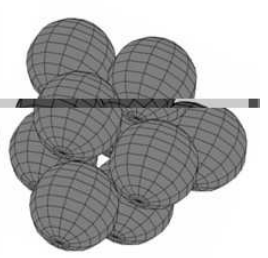

(c)
FIG. 4: Comparison for $N=9$ between the packing given by (a) experiments, (b) simulations and (c) model.

modes for the packing, we obtain therefore that there exists a unique mode of rearrangement for all particles which are non-rattlers. In experiments, the degeneracy in the circle packing problem is chosen by additional information: for example in [1], because the particles are charged only on the side exposed to the water, there is a weak dipolar repulsive force between the particles which breaks the degeneracy [10] 14].

This result implies that there is only a single set of $\left\{F_{i}\right\}$ that is consistent with all the constraints. This mode is independent of surface energies, and depends only on geometry. Capillarity does enter into the problem in relating the force $F_{i}$ to the displacement $\delta r_{i}$ of the $i^{t h}$ sphere. If radius of curvature of the droplet changes from $R$ to $R+\delta R$ then volume conservation of the droplet implies that $\delta r_{i}$ and $\delta R$ are related through $A \sum_{i} \delta r_{i}+$ $\left(4 \pi R^{2}-N A\right) \delta R=0$, where $A$ is the wetted area of the particles. The capillary force $F_{i}$ is then given by

$$
F_{i}=-2 \pi \gamma_{D} \cos \beta\left(\delta r_{i}+\frac{A \cos \beta}{4 \pi R^{2}-N A} \sum_{i} \delta r_{i}\right),
$$

where $\alpha$ is the dry angle of the particle on the critical packing, $\theta$ the equilibrium contact angle, and $\beta=\alpha-\theta$. This formula is asymptotically valid in the limit $\delta V \rightarrow 0$ so that deviations from a spherical cap droplet are small.

The above results apply just below $V_{c}$. However, the general principle can be iteratively applied below the critical volume: starting from the spherical packing, we decrease the droplet volume by small increments and, assuming equation (3) continues to hold, we calculate the corresponding incremental particle rearrangement consistent with all the constraints. At each step in the iteration it is necessary to recompute the mode $\left\{F_{i}\right\}$; for every packing there is a unique choice that is consistent with the constraints. This process iterates until the final equilibrium configuration is reached. During the process the packing changes substantially from the initial disk packing - typically multiple new contacts are added.

The results of the model are displayed in Table 1 and in Figure 4 The model reproduces accurately the final experimental packings for $N \leq 6$ and $9 \leq N \leq 14$; in particular, the non-convex packing for $N=11$ is well predicted by the model. There are small differences in the final packings for $N=7$ and 8, likely arising from deviations of the capillary force-particle displacement re- 


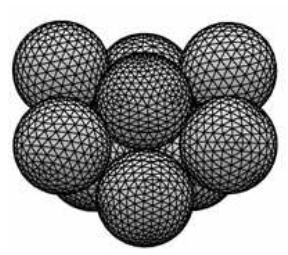

(a)

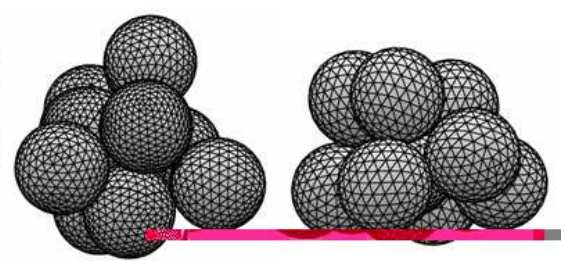

(c)
FIG. 5: Final configuration of 9 spheres with different wetting conditions: on the droplet, $n_{1}$ spheres have a contact angle of $160^{\circ}$ and $n_{2}=9-n_{1}$ spheres have a contact angle of $20^{\circ}$. (a) cases $\left\{n_{1}, n_{2}\right\}=\{2,7\}$ and $\{4,5\}, \mathcal{M}=27.706$; (b) case $\left\{n_{1}, n_{2}\right\}=\{6,3\}, \mathcal{M}=29.780 ;\left(\right.$ c) case $\left\{n_{1}, n_{2}\right\}=\{8,1\}$, $\mathcal{M}=30.754$ (units of the particle radius).

lationship from the linear law (3). Overall, the model leads to a dimensionless error in second moment with the experimental packings of $1.6 \%$ versus $2.6 \%$ for the minimum second moment criterion. It should be emphasized that the computational cost of the new algorithm is orders of magnitude slower than that of a full simulation. To our surprise the final packing can be computed quite accurately without ever knowing the shape of the liquid surface during the packing process!

Finally, we remark on the minimal moment criterion itself: our results suggest that the drying influences the final packings only through (a) enforcing the initial disk packing at the critical volume; and (b) through equation (3), relating the $F_{i}$ to the particle displacements. It is probably not coincidental that (up to prefactors) equation (3) is similar to the force-displacement relation $F_{i}=-\partial_{i} \mathcal{M} \sim c_{1} \delta r_{i}+c_{2} \sum_{i} \delta r_{i}$, for the minimal moment criterion. We believe that this explains the similarity between the observed structures and those minimizing the second moment.

The calculations presented herein suggest that the unique packings observed by Manoharan et. al. arise because (i) the initial circular packing is unique for the regime they explored, except for $N=15$; and (ii) the subsequent evolution of the particles is so highly constrained that there is only one final packing that is consistent with the constraints.

This suggests that the only way to generate different packings is to modify the circle packing at the critical volume. This can be easily modified by choosing particles with differing sizes or wettabilities (thus creating circles of different sizes). We have run simulations where particles on a given droplet possess different contact angles. Figure 5 shows three cases of $N=9$ particles with contact angles of either $160^{\circ}$ or $20^{\circ}$. The three packings and their second moment differ significantly from Fig. 4

In summary, we have presented a numerical and theoretical study of the packing selection problem of Manoharan et al. [1]. The selection of a unique packing was found to arise almost entirely from geometrical constraints during the drying process and a procedure was proposed to generate different packings. This could be extended to characterize all admissible evaporation-driven packings of colloidal spheres.

We thank Henry Chen, Vinothan Manoharan, David Pine and Howard Stone for useful discussions. We also thank Ken Brakke for his help with The Surface Evolver. We gratefully acknowledge the Office of Naval Research and the Harvard MRSEC for supporting this research.
[1] V.N. Manoharan, M.T. Elsesser, and D.J. Pine (2003) Science 301, 483-487.

[2] Sloane N.J.A., Hardin R.H., Duff T.S. \& Conway J.H. (1995) Disc. Comp. Geom. 14 237-259. Packings available at www.research.att.com/ njas/packings/

[3] Kralchevsky P.A. \& Denkov N.D. (2001) Curr. Opin. Colloid. Int. Sci. 6 383-401.

[4] Brakke K. (1992) Exp Math. 1 141-165.

[5] Melnyk T.W., Knop O. \& Smith W.R. (1977) Can. J. Chem. 55 1745-1761.

[6] Clare B.W. \& Kepert D.L. (1986) Proc. Roy. Soc. A 405 329-344.

[7] Sloane N.J.A. with the collaboration of Hardin R.H., Smith W.D. and others, Tables of Spherical Codes, published electronically at www.research.att.com/ njas/packings/

[8] Kottwitz D.A. (1991) Acta. Cryst. A 47 158-165.

[9] Tkachenko A.V. and Witten T.A. (1999) Phys. Rev. E 60 687-696.

[10] Pieranski P. (1980) Phys. Rev. Lett. 45 569-572.

[11] Hurd A.J. (1985) J. Phys. A: Math. Gen. 18 L1055-1060.
[12] We have used potentials ranging from exponential $U_{R} \sim$ $a \exp p\left(1-d / d_{0}\right)$, to power law, $U_{R} \sim a\left(d_{0} / d\right)^{p}$, where $d\left(d_{0}\right)$ is the distance between two particles (diameter of a particle). The results are insensitive to the exact functional form of $U_{R}$.

[13] Since $\sum_{i} \mathbf{F}_{i}=\mathbf{0}$, we get necessarily that $n_{f} \leq N-3$.

[14] The strength of the dipolar interaction on $n_{c}$ contacts is about $U_{\text {elec }} \sim n_{c} p^{2} / \epsilon_{r} \epsilon_{0} a^{3}$ with a dipole moment $p \sim \sigma a^{2} / \kappa \sqrt{\epsilon_{r}}[11]$ where $a$ is the particle size, $\epsilon_{r}$ the dielectric constant of water and $\sigma$ the surface charge of the colloids with zeta potential $\zeta$ and screening length $\kappa^{-1}$, $\sigma \sim \zeta \epsilon_{0} \epsilon_{r} \kappa$; therefore $U_{\text {elec }} \sim n_{c} \zeta^{2} \epsilon_{0} a$. By comparison, the change in surface energies $\delta U_{\Sigma}$ due to a translation of order $\delta a$ of the contact line of each particle and the creation of menisci is $\delta U_{\Sigma} \sim N \gamma_{D} a \delta a$. Since $n_{c} \sim 2 N$, and with the values $\zeta \approx 100 \mathrm{mV}, a \approx 1 \mu \mathrm{m}$ and $\gamma_{D} \approx 10^{-2}$ N.m ${ }^{-1}$, we get that $U_{\text {elec }} \geq U_{\Sigma}$ when $\delta a \leq 10^{-5} a \ll 1$ $\mathrm{nm}$. Hence, as soon as menisci appear, the dipolar repulsion appears only as a secondary minimization problem, governing the location of particles after surface energies are minimized. 